\title{
EL YO DIVIDIDO EN LA LENGUA Y EN LOS SUEÑOS
}

\section{THE DIVIDED SELF IN LANGUAGE AND IN DREAMS}

\author{
Roberto Carlos Arias Oliveira \\ Doctor en Lingüística, Bruselas, Bélgica
}

Cómo referenciar este artículo/How to reference this article:

Arias Oliveira, R. C. (2019). El Yo Dividido en la Lengua y en los Sueños. Revista de Psicoterapia, 30(112), 131-145. https://doi.org/10.33898/rdp.v30i112.257

\begin{abstract}
Resumen
Son muchos los autores que han observado que, en los sueños, es frecuente que el yo aparezca representado en más de un participante. La lingüística, por su parte, también ha llamado la atención sobre distintos patrones de la lengua en los que el yo aparece dividido en más de una instancia. Ante el paralelismo de ambos fenómenos, cabe preguntarse si existe un vínculo entre ellos. En este artículo, como lingüista, partiré de lo que puede observarse a través de la lengua para intentar elucidar un modelo de representación del yo en los sueños. A través de una serie de ejemplos se verá que, en efecto, en los sueños se observan patrones que reflejan las mismas tendencias que revela la lengua. El trabajo pretende así perfilar una herramienta que pueda servir de complemento a los diferentes enfoques y métodos existentes para abordar los sueños.

Palabras clave: Sueños, lengua y sueños, yo dividido, representación del yo, metáfora
\end{abstract}

\begin{abstract}
:
It has been observed by numerous scholars that the self can be represented in dreams through more than one character. At the same time, linguists have identified a series of patterns in language in which the self is divided into differententities. In view of the conspicuous parallelism between both phenomena, it seemsjustified to ask whether they are related. As a linguist, in this article I will use the patterns that can be observed in language to try to flesh out a model of how the self is represented in dreams. By means of several examples, it will be shown that dreams exhibit patterns that mirror what can be detected in language. The aim of the paper is to sketch out a tool that can be used when working with dreams, thus complementing existing methods and approaches.
\end{abstract}

Keywords: Dreams, language and dreams, split self, representation of the self, metaphor 


\section{Introducción: de la Lengua al Sueño}

Desde clásicos como Fromm (1957), Jung (1916/2001) o el propio Freud (1900/1991) a las obras de psicoterapeutas contemporáneos que trabajan con sueños (Cushway y Sewell , 2013; Montangero, 2013), la observación de que el yo puede aparecer representado en más de un personaje de un sueño es recurrente en la bibliografía. Se acepta así ampliamente que la persona que sueña puede desempeñar a la vez más de un "papel" en las escenas oníricas. Por su parte, la lingüística ha observado que, en la lengua, es frecuente que el yo aparezca dividido en más de una instancia, y se ha llamado la atención sobre algunos modelos recurrentes de yo dividido comunes a idiomas muy diversos. Ante el paralelismo entre una y otra idea, cabe plantearse si puede existir una relación entre lo que se manifiesta en la lengua y lo que se manifiesta en los sueños. De hecho, si consideramos que tanto la lengua como los sueños hunden sus raíces en el inconsciente, resulta difícil pensar que pueda tratarse de fenómenos independientes sin relación entre sí.

Desde la lingüística, en este artículo se parte de los fenómenos que se pueden observar en la lengua para ver si estos permiten elucidar un modelo de la representación del yo en los sueños. El objetivo es perfilar así una herramienta que pueda servir de complemento a los instrumentos y métodos existentes para abordar los sueños, tanto para la propia introspección como para el trabajo con sueños en diferentes contextos, como puede ser la terapia. El hecho de que el trabajo esté elaborado por un lingüista, y no por un terapeuta, puede sin duda representar una dificultad para alcanzar este objetivo; no obstante, en mi opinión, conlleva también la ventaja de aportar una perspectiva complementaria que se apoya sólidamente en otra manifestación humana.

La intuición de que existen vínculos entre la lengua y los sueños no es nueva, y se ha plasmado ya de múltiples formas en la bibliografía (Freud, 1900/1991; Foulkes, 1978; Fromm, 1957; Montangero, 2007, 2013)1', aunque, posiblemente, ninguna obra se ha ocupado directamente de los paralelismos en la división del yo. Por lo demás, la razón de que haya posibles vínculos entre lengua y sueños puede buscarse, entre otras cosas, en el papel que el pensamiento inconsciente desempeña en ambas. Así, en la lengua salen a relucir aspectos que van más allá de lo puramente lingüístico, y en ella quedan reflejados fenómenos más generales del pensamiento humano que, a menudo, no percibe la mente consciente ${ }^{2}$ (Lakoff y Johnson, 1999).

Se asume que los sueños constituyen una forma de pensamiento, la que corresponde al modo de funcionamiento más relajado de la mente (Hartmann, 2007, 2010; Montangero, 2007, 2013). En este sentido, por mucho que su contenido pueda resultar extraño a la mente despierta, no hay motivo para considerar que no tengan sentido. No obstante, la dificultad se plantea sin duda a la hora de estudiar dicho contenido del modo en que se va a hacer en este trabajo, ya que las observaciones irán vinculadas necesariamente a lo que se conoce como interpretación, y difícilmente se puede demostrar que la interpretación es correcta. Aun así, se considera que, dentro de los fines descritos, puede ser igualmente útil trabajar con explicacio- 
nes plausibles que ayuden a observar patrones recurrentes.

Un fenómeno en el que ya se han abordado los paralelismos entre lengua y sueño es el conocido como metáfora conceptual (Lakfoff y Johnson, 1999; Soriano, 2012). A diferencia de lo que se entiende habitualmente por metáfora, la metáfora conceptual no designa expresiones lingüísticas concretas, sino correspondencias sistemáticas entre dominios más amplios. La dinámica es normalmente la siguiente: para comprender y manejar conceptos abstractos, proyectamos uno de los dominios (el dominio origen), que es más concreto, sobre el otro (el dominio meta), que suele ser de naturaleza más abstracta. Estas proyecciones se plasman en la lengua, pero tienen lugar a nivel de pensamiento. Un ejemplo es una metáfora muy común en la lengua que podemos formular ${ }^{3}$ como SABER ES VER. Esta metáfora se plasma en expresiones como: ¿Ves lo que quiero decir?; ojos que no ven, corazón que no siente; por fin se le ha caído la venda de los ojos. Metáforas como esta pueden ser muy relevantes en la evolución lingüística. Por ejemplo, el verbo alemán wissen, que significa "saber", está emparentado con el verbo latino videre, que significaba "ver", por lo que en algún punto de la evolución lingüística la metáfora SABER ES VER dio lugar a un cambio de significado. Los ejemplos de este tipo en la lengua son numerosos.

Se ha observado (Lakoff, 1997a, 1998; Bolognesi y Bichisecchi, 2014; Malinowski y Horton, 2015; Kramer, 2017; Hartmann, 2007; Montangero, 2007; Enyon, 2002) $)^{4}$ que la metáfora conceptual puede desempeñar un papel clave en los sueños. Así, a través de SABER ES VER, un sueño de ceguera puede plasmar el temor de una persona a no saber lo suficiente o a ignorar algo ${ }^{5}$. De este modo, se ha reconocido en la metáfora un potente instrumento para acceder al contenido de los sueños. En las páginas que siguen (apartados 3 a 6), se utilizará a menudo de la metáfora como medio con el que abordar, a partir de la lengua, los sueños que se expondrán. Antes, no obstante, será necesario exponer algunos aspectos esenciales de la división del yo en la lengua.

\section{EI Yo Dividido en la Lengua}

En la lengua no es raro que un individuo o lo que, de manera general, podemos denominar su psique, aparezca dividido en dos o más entidades diferentes. Es frecuente hablar de subject, instancia que recoge esencialmente la conciencia en su calidad de ente experimentador, incluyendo la racionalidad y el juicio; y de self, que suele abarcar todo el resto, incluyendo el cuerpo, la parte "irracional" de la psique y el comportamiento social (Lakoff, 1997; Lakoff y Johnson, 1999; Talmy, 2000, 2015; Haimann, 1995) ${ }^{6}$. A partir de estas nociones, la bibliografía (Domaradzki, 2011; Hirose, 2014) ha identificado una serie de modelos recurrentes comunes a idiomas diversos, como el árabe y el japonés respectivamente, expresables por lo general en forma de metáfora, por ejemplo:

- El SELF ES UN CONTENEDOR PARA El SUBJECT: Por virtud de esta metáfora, el subject ejerce control sobre el self cuando está "dentro" de él, como en 
Estaba fuera de sí, o Vuelve en ti antes de que sea demasiado tarde.

- El SUBJECT Y EL SELF SON INDIVIDUOS QUE INTERACCIONAN: A través de esta metáfora, el subject y el self pueden aparecer como adversarios (Está luchando consigo mismo) o simples interlocutores (Se convenció a sí mismo de que era lo mejor).

En lugar de exponer los diferentes modelos que ha resaltado la bibliografía, se señalan aquí únicamente dos tendencias generales que están muy presentes en la configuración de estos modelos ${ }^{7}$. Si bien podrían estar interrelacionadas, se pueden tratar por separado:

A. La autonomía de un "núcleo" de facultades superiores

El primero de estos fenómenos es la autonomía que se atribuye a un pequeño núcleo de facultades superiores que abarca ante todo la conciencia, el juicio y, posiblemente, la volición. Estas facultades conforman una entidad que tiene la capacidad de "independizarse" del resto de la persona y juzgarla. Es lo que se ve en casos como los siguientes:

No está contento consigo mismo.

Cuando me miro, veo a alguien que no me gusta.

Esta entidad, de hecho, también puede "proyectarse" en terceras personas. Esto es lo que sucede, por ejemplo, al usar expresiones como las siguientes:

Ponte en mi lugar y dime qué harías.

Si yo fuera tú, no hablaría con alguien como yo.

Obsérvese que a estos casos subyace la idea de que la conciencia y el juicio de un individuo pueden "proyectarse" o "superponerse" al conjunto de la persona de otro individuo, manteniendo la capacidad de emitir juicios.

B. El control de una instancia sobre otra

El otro fenómeno consiste en representar el comportamiento del yo como la interacción entre dos instancias. En estos casos, la división del yo va asociada al control que una parte del yo ejerce o debe ejercer sobre la otra. Algunos ejemplos serían los siguientes:

Le cuesta mucho trabajo controlarse cuando bebe.

No tiene las riendas de sí mismo.

Aunque las dos instancias en las que se divide el yo en estos casos no están claramente delimitadas, se pueden caracterizar esquemáticamente de la siguiente forma:

1. A una se le atribuye la capacidad de ejercer autoridad sobre la otra. Por lo general, se considera que es acorde con los valores sociales y con un sentido de la responsabilidad. A ella me referiré en adelante como YO ${ }^{1}$.

2. La otra instancia debe ser controlada, especialmente en situaciones en las que es preciso cumplir con los valores sociales. Por lo general, se le atribuye un carácter más irracional. A ella me referiré en adelante como $\mathrm{YO}^{2}$.

Numerosos procesos psicológicos se representan en la lengua como una 
interacción entre el $\mathrm{YO}^{1}$ y el $\mathrm{YO}^{2}$, normalmente en una lucha por el control. Veamos algunos ejemplos:

Estuve durante toda la cena conteniéndome: el $\mathrm{YO}^{2}$ intenta irrumpir y manifestarse, pero el $\mathrm{YO}^{1}$ consigue mantener el control sobre él.

Me dejé llevar por mis impulsos: aquí se representa un escenario figurado en el que el $\mathrm{YO}^{1}$ acepta seguir los dictados del $\mathrm{YO}^{2}$.

Me he tenido que obligar a levantarme temprano: $\mathrm{el}^{\mathrm{YO}^{1}}$ asume la tarea de "dirigir" a un $\mathrm{YO}^{2}$ que hubiera preferido seguir durmiendo.

Tal vez sea importante recordar que no se está afirmando que la psique se divida realmente en dos o varias instancias, ni que los fenómenos aquí expuestos correspondan a la "verdad" de la psicología humana. Lo que he detallado aquí son formas inconscientes de representarnos a nosotros mismos y de capturar la dinámica de algunos procesos psíquicos cotidianos, tal y como quedan plasmados en la lengua.

A continuación, se propone ver si estos dos fenómenos permiten explicar la representación del yo en los sueños. Para ello, se expondrá y analizará una serie de sueños reales de diferentes personas.

\section{El Yo Frente al Yo Observador}

En este apartado se comenzará el análisis con tres ejemplos breves y simples.

\section{Ejemplo 1: Sara duplicada}

Este primer ejemplo es un sueño que tuvo llamada Sara.

\section{Sueño de las dos Saras}

Sara está conversando con dos personas. Una de las personas es su novio, la otra es una chica. En un momento dado, la chica se dirige a Sara y le pregunta: “¿Por qué no le dices eso tú?” Sara se da cuenta entonces de que la otra chica también es ella.

Sara aparece aquí representada en dos participantes distintos. El primero es el que podríamos designar como la propia Sara en su calidad de observadora, es decir, quien está viviendo el sueño y mantiene la conciencia de ser Sara. A esta instancia se la denominará YO OBSERVADOR. El segundo participante es la otra chica que está igualmente presente en la conversación, y que Sara ve "desde fuera". Se puede decir que esta figura corresponde a un yo externalizado de Sara. A esta forma de representación externalizada será referida en adelante por lo general como yo.

Al final de este corto sueño, Sara cobra conciencia de que la otra persona también es ella, pero esta toma de conciencia no tiene por qué tener lugar. De hecho, en los casos que veremos a continuación, en ningún momento la persona que sueña es consciente de que las otras personas en las que aparece representada son también ella misma. 


\section{Ejemplo 2: emociones paralelas}

Se describe un sueño autorreferencial:

Sueño del teléfono en la mesita de noche

Mi padre está durmiendo. Mi tía Amalia entra en la habitación y se pone a hablar por un teléfono que está en la mesita de noche, al lado de la cama. En su conversación habla de "ir a Lucena". A mí me irrita ver cómo está molestando el sueño de mi padre. Yo me enfado con ella. Mi padre, furibundo, desiste finalmente de su intento de dormir.

Algunos elementos del contexto se hacen aquí necesarios: cuando se tuvo el sueño, el protagonista vivía en un piso compartido; la noche anterior, se había acostado tarde y, por la mañana, una compañera de piso se puso a hablar con una amiga (muy fuerte, en la percepción del protagonista) al lado de la habitación. A esta molestia se sumaba otro incordio, y es que por la ventana entraba ya demasiada luz como para permitir dormir bien. De hecho, la falta de persianas y el ruido de los compañeros, en general, hacía que se alimentara desde hacía un tiempo la idea de mudarse de aquel piso.

Como puede verse, el yo aparece representado aquí en la figura de "Mi padre", que está durmiendo. "Mi tía Amalia", que entra y se pone a hablar desconsideradamente junto a la cama, representa a la compañera de piso. Hay que decir que Amalia se caracteriza por ser muy parlanchina y por hablar muy fuerte, por lo que, aunque obviamente se trata de una hipérbole, la elección del personaje femenino es del todo menos azarosa. Por lo demás, todo apunta a que Lucena es una referencia a la molesta $l u z$ que entraba a raudales por la ventana ${ }^{8}$.

A pesar de su simpleza, hay dos aspectos particularmente interesantes en este sueño. Uno es cómo las instancias del yo reaccionan a un mismo acontecimiento e interactúan con un tercer personaje; así, el YO OBSERVADOR, se enfada, mientras que el yo se muestra furibundo. Más adelante, en un caso más complejo, volveré a las emociones que se atribuyen a las instancias del yo.

El otro aspecto de interés es el hecho de que el yo aparezca representado precisamente a través de "Mi padre". Con ello, la figura que encarna una instancia del yo está unida al mí en la realidad por un vínculo de parentesco. Este fenómeno, que será encontrado más adelante, puede expresarse como metáfora, adoptando la siguiente formulación: UN VÍNCULO INTRAPSÍQUICO ES UN VÍNCULO FAMILIAR.

\section{Ejemplo 3: modelo A y modelo B}

El tercer ejemplo es, nuevamente, un sueño de Sara. Se desarrolla en dos partes:

Parte I:

$$
\text { Sueño de "Twin Peaks" }
$$

Sara va caminando por la playa. Se encuentra con una especie de gran 
ballena. Sara siente deseos de matarla o de quitarla de en medio, aunque la ballena no está haciendo daño alguno ni es agresiva.

Parte II:

Está con Leo y Shelley, dos personajes de la serie Twin Peaks (Lynch y Frost, 1990). Leo y Shelley se muestran enamorados, y Sara piensa: "Se han reconciliado". En alguna parte hay bicho, quizá un insecto. Sara lo observa, esperando a que muera. Pero el bicho no muere, sino que va a atacarla a ella.

Cuando tuvo el sueño, Sara estaba preocupada por su relación amorosa debido a la actitud que observaba en su novio, y sus inquietudes o temores quedan plasmados en ambas partes en la presencia indeseable de animales: en la primera parte es un animal de gran tamaño (es decir, difícil de ignorar), mientras que en la segunda parte es un insecto (es decir, un tipo de animal que fácilmente se antoja desagradable). En ambos casos, Sara espera su muerte, o sea, su desaparición.

Se puede asumir que ambas partes son reelaboraciones de una misma idea, pero cada una de las reelaboraciones se centra en aspectos distintos, lo que implica distintas representaciones de la propia Sara. En la primera parte, Sara solo está presente a través del Yo OBSERVADOR. Más interesante es la representación del yo en la segunda parte, en la que hacen su aparición los dos personajes de Twin Peaks (Lynch y Frost, 1990). Sara es gran admiradora de esta serie americana de los noventa, y ha visto varias veces todos los capítulos. Cuando le pedí que me dijera quiénes son Leo y Shelley, la propia explicación fue de por sí elocuente: se trata de una pareja que nunca podría ser feliz, se engañan mutuamente y él intenta matarla en una ocasión. De forma hiperbólica ${ }^{9}$, pues, Sara está representando su propia relación a través de esta pareja de ficción. El propio hecho de que la pareja aparezca de forma armoniosa se puede considerar inquietante: por el contexto, que Sara conoce bien, se sabe que no van a poder ser felices, y que la aparente armonía es falsa. De manera redundante, este hecho también está recogido en la presencia indeseable del insecto. Al proyectar a la pareja que conforman ella misma y su novio en la pareja Shelley-Leo, Sara está representándose a sí misma a través de Shelley: Shelley es, pues, su yo. Con ello está atribuyéndose a sí misma algunos rasgos relevantes de este personaje, como el encontrarse en una relación infeliz. A la vez, está atribuyendo a su novio los rasgos negativos de Leo, como el tener un marcado lado oscuro. Aunque se trata de una representación a todas luces tendenciosa y exagerada, se puede apreciar que la selección de personajes no es en absoluto fruto del azar.

Se puede observar que cada una de las partes de este sueño muestra un modelo de representación del yo diferente:

- En la primera parte, Sara solo está presente a través del yo OBSERVADOR. Se modelo referirá en adelante como modelo $A$.

- En la segunda parte, Sara está presente a través del Yo OBSERVADOR y de un yo externalizado. A este modelo se referirá en adelante como modelo B. Es, por cierto, el modelo que se ha visto también en el "Sueño de las dos Saras" 
y en el "Sueño del teléfono en la mesita de noche".

\section{EI Yo Desdoblado}

El siguiente ejemplo es un sueño que tuvo Susanne. Con él se introducirá un nuevo modelo de representación del yo.

Sueño de la chica de los cuchillos

Susanne y su novio están siendo observados, a través de la ventana, por dos mujeres que se encuentran en el edificio de enfrente. Después de estar un rato mirando desde dos ventanas separadas, las mujeres cruzan de pronto el espacio que hay entre ambos edificios, como por una pasarela o simplemente por el aire. Una de las dos mujeres es una chica joven, mientras que la otra es de más edad. Una vez que está en el piso, la chica joven se vuelve peligrosa y agresiva. Tiene cuchillos en las manos. La mujer mayor, que muestra un comportamiento más calmado, intenta por su parte apaciguar la situación y tranquilizar a Susanne, diciéndole que tiene que hablar con su novio y explicárselo todo.

Algunas aclaraciones del contexto se hacen necesarias antes de abordar el sueño. Cuando lo tuvo, Susanne se encontraba en su casa con su novio, con el que estaba comenzando una relación. Por experiencias pasadas, Susanne, que se define como una chica sensible e inteligente, tenía miedo de sacar su lado más agresivo y destructivo en la incipiente relación. Es este miedo el que sale a relucir en el sueño.

Pero ¿por qué dos mujeres? Una característica destacable de la pareja que ambas conforman es el hecho de que los atributos psíquicos de una y de otra estén asignados de manera complementaria:

a) A una se le atribuye un comportamiento más calmado, así como la capacidad de controlar la situación y ejercer cierta autoridad.

b) A la otra se le asigna un carácter más irracional y, en la situación en cuestión, se considera conveniente que sea controlada.

Estos rasgos de una y de otra concuerdan asimismo con la diferencia de edad que se les atribuye, ya que es común aceptar que a mayor edad corresponden un mayor sentido de la responsabilidad y una mayor autoridad.

Como puede verse, existe un paralelismo notable entre las características de estos personajes y los rasgos que muestran el $\mathrm{YO}^{1}$ y el $\mathrm{YO}^{2}$ en la lengua. En el apartado 2 , se veían dos instancias en una relación asimétrica, en la que solo a una (el yo $\left.{ }^{1}\right)$ se asigna la responsabilidad de los actos y la capacidad de controlar mientras la otra $\left(\mathrm{el} \mathrm{YO}^{2}\right)$ necesita ser controlada o tutelada. Por estos importantes paralelismos, y también por el hecho de que ambas figuras, a pesar de las disparidades, actúen de manera conjunta (las dos provienen del mismo lugar e irrumpen juntas en el piso), parece probable que ambas representen a la propia Susanne. Para reflejar la analogía que se da con la lengua, los personajes del sueño también serán denominados como $\mathrm{YO}^{1}$ y YO$^{2}$ : la mujer que muestra un temperamento más calmado es el $\mathrm{YO}^{1}$, mientras que la chica de los cuchillos es el $\mathrm{YO}^{2}$. 
El sueño capta así de manera altamente expresiva las inquietudes de Susanne. El proceso psíquico en cuestión queda representado mediante la actuación descontrolada de un $\mathrm{YO}^{2}$ capaz de hacer estragos si no se le controla. El modelo de representación del yo que se da en este sueño es distinto a los dos modelos que se han visto hasta ahora. Aquí la persona que sueña está presente a través del yo OBSERVADOR y de otras dos personas, con lo que Susanne estaría representada en un total de tres instancias. ¿Cómo se explica esta nueva multiplicidad de figuras? Hay aquí dos procesos operando en la representación del yo, y ambos procesos muestran paralelismos con lo descrito en el apartado 2:

1) En primer lugar, hay una externalización, que provoca que Susanne esté presente tanto en el YO OBSERVADOR como en otras figuras de la escena.

2) En segundo lugar, se produce además una representación dividida del yo externalizado, que está así repartido en dos figuras: el $\mathrm{YO}^{1}$ y el $\mathrm{YO}^{2}$.

A este tercer modelo de representación del yo me referiré como modelo $C$. En el Gráfico 1 se muestran de manera simplificada los tres modelos ${ }^{10}$.

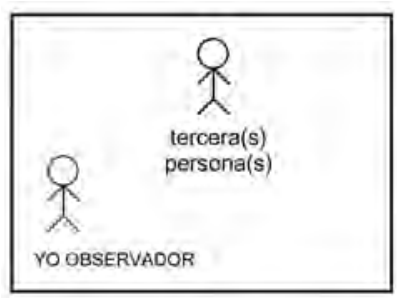

Modelo A

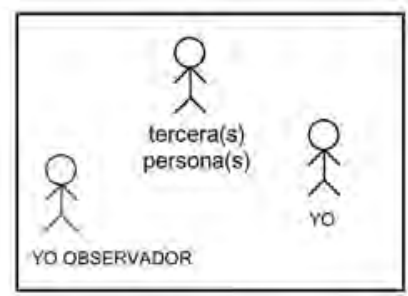

Modelo B

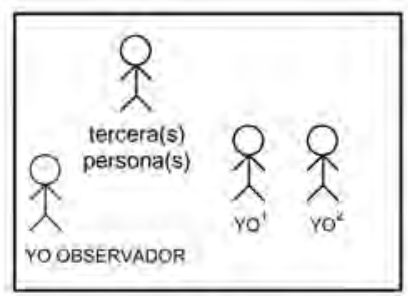

Modelo C

Gráfico 1. Modelo C.

\section{A cada instancia lo que le toca}

A continuación, se expondrá un último ejemplo, nuevamente un sueño autorreferencial. Aunque fugazmente, este último ejemplo permitirá abordar cuestiones como las características psíquicas atribuibles a cada instancia del yo y el modo en que estas pueden interactuar entre sí.

\section{Sueño de la agresión}

Estoy en un piso [indeterminado] y veo (como a través de la mirilla de la puerta) cómo llega al rellano mi tío Joaquín. Yo abro la puerta para dejarlo entrar $\mathrm{y}$, entonces, le agredo. Le pego con una furia inquebrantable. Después de la agresión, siento dudas y no sé muy bien cómo actuar ante el resto de la familia. Estoy con mi padre y mi tío Rodrigo (este último aparece con un abrigo largo negro) y no sé si decírselo. Ignoro si pueden haberse enterado, aunque me parece muy posible que estén ya al tanto. Mi padre está serio, y mi tío Rodrigo se muestra abatido, pero ninguno de los dos menciona el tema. 
Por su configuración y por los elementos de violencia (Oliveira, en prensa) ${ }^{11}$, el sueño parece remitir a una fuerte discusión que se había tenido poco tiempo antes con quien por entonces era la pareja, Miriam, y que dejó una profunda huella en el estado de ánimo del protagonista. Algunos detalles son relevantes: cuando el autor regresaba de un viaje, Miriam estaba esperándolo en el piso del protagonista para hablar él, pero la conversación no tardó en degenerar en una acalorada discusión, que fue vivida como un repentino asalto, debido a la sorpresa que causó. El incidente dejó abatido al protagonista, pero lo cierto es que no se prolongó tanto como, dadas las circunstancias, el autor se temía, y pudo zanjarse con relativa facilidad, tras lo cual el autor refirió no darle más vueltas al asunto.

Para mayor comodidad en el análisis, se divide el sueño en dos mitades o escenas: la primera abarcará el inicio del sueño y la paliza, y la segunda, lo que ocurre después de la paliza. Como puede verse, el inicio de la primera escena reconstruye el contexto de la mencionada discusión de forma más o menos fidedigna: alguien, concretamente un hombre, llega a una vivienda sin saber que le espera una agresión. Una metáfora formulable como AGRESIVIDAD VERBAL ES AGRESIVIDAD FíSICA explica que una discusión pueda estar representada a través de una paliza. La agresión refleja la agresividad que se percibió en aquel momento y, obviamente, está representada desde una óptica subjetiva e hiperbólica. Hay algo que, no obstante, difícilmente se puede pasar por alto: quien perpetra la agresión es el Yo OBSERVADOR. La víctima es Joaquín, que no tiene nada que ver en el contexto de la discusión con Miriam. ¿A qué se debe, pues, esta extraña configuración de personajes?

Por su papel como víctima de la agresión, y también por el hecho de ser un hombre y ser pariente (a través de UN VíNCULO INTRAPSÍQUICO ES UN VÍNCULO FAMILIAR), parece plausible que Joaquín está representando al si mismo en la discusión. Joaquín, en la realidad, había sufrido también problemas de pareja, por lo que la elección del "actor" tampoco obedece al azar en este caso. No obstante, falta por explicar el factor más desconcertante de la escena: por qué es el YO OBSERVADOR el que perpetra la agresión sobre el Yo. En un principio, podrían considerarse al menos las posibilidades siguientes:

a) Que la escena esté narrada desde una supuesta perspectiva de Miriam: ella es quien espera en el piso y, cuando el protagonista entra, perpetra lo que se representa como una agresión. En este caso, el YO OBSERVADOR correspondería a Miriam.

b) Que la escena no esté narrada ni desde la perspectiva de Miriam ni desde otra perspectiva diferente a la del autor, sino que el autor se asigne a si mismo tanto el papel de agresor como el papel de agredido.

Antes de intentar decantarse por una de estas opciones, se considera conveniente echar un vistazo a la segunda escena. Una vez perpetrada la agresión, aparecen el padre y el tío Rodrigo. Ambos conforman una nueva pareja que entra en escena de manera conjunta y en la que los componentes muestran una diferencia 
de edad relevante (Rodrigo es hermano menor del padre y, por la personalidad de ambos, los papeles siempre han estado muy claros). Todo apunta a que estamos así nuevamente ante un doblete de $\mathrm{YO}^{1} \mathrm{y} \mathrm{YO}^{2}$, unido por la metáfora UN víNCULO INTRAPSÍQUICO ES UN VÍNCULO FAMILIAR. Así, la segunda escena está articulada siguiendo el modelo $\mathrm{C}$ de representación del yo.

Por la mencionada diferencia de edad, parece lógico asumir que a el padre le corresponde el papel de $\mathrm{YO}^{1}$ y al tío Rodrigo, el de $\mathrm{YO}^{2}$, pero hay asimismo otras razones que hablan a favor de esta distribución. Un rasgo propio de $\mathrm{YO}^{2}$ que presenta el tío en el sueño es que él manifiesta más claramente sentimientos (está abatido), como cabe esperar en la instancia a la que se atribuye lo irracional, como las emociones; por cierto, va además vestido de negro, lo que es señal comúnmente aceptada de tristeza. El padre, por su parte, muestra actitud seria, lo que está mucho más en consonancia con la naturaleza racional del $\mathrm{YO}^{1}$, tal y como se describió en el apartado 2.

El comportamiento de ambos hombres refleja la propia actitud del protagonista aquella noche después de la discusión: se quedó serio y abatido, aunque prefiere dejar las cosas como están y no darle más vueltas al asunto. El yo OBSERVADOR en esta escena añade otro rasgo más a la descripción del estado anímico del protagonista tras la discusión: siente dudas y no sabe cómo actuar. Así, un punto especialmente interesante de la representación es que cada uno de los componentes que conforman lo que es un complejo paisaje psíquico está atribuido a una instancia del yo diferente: la primera no sabe cómo actuar, la segunda está seria y la tercera, abatida. El modo en que se distribuyen estas manifestaciones psíquicas no es arbitrario, sino se ajusta al reparto de competencias que también se observa en la lengua, ya que cada una de las manifestaciones es relativamente acorde con la naturaleza de la instancia en cuestión. Así, el Yo OBSERVADOR, al que se le asignan la conciencia y el juicio, alberga dudas acerca de cómo actuar; el $\mathrm{YO}^{1}$, relacionado con la racionalidad y el control de la situación, muestra seriedad; por último, el $\mathrm{YO}^{2}$, al que se atribuye la parte más irracional, como las emociones, está abatido. Este reparto complementario de atributos psíquicos queda representado en la tabla 1.

Tabla 1. Reparto de atributos psíquicos.

\begin{tabular}{lll}
\hline YO OBSERVADOR & Conciencia y juicio & Dudas sobre cómo actuar \\
\hline $\mathrm{YO}^{1}$ & Control & Seriedad \\
\hline $\mathrm{YO}^{2}$ & Emociones & Abatimiento \\
\hline
\end{tabular}

\section{¿Reflexividad Onírica?}

En una oración como Juan golpeó a Manolo, Juan es quien golpea (el agente) y Manolo, quien es golpeado (el paciente). Si digo Juan se golpeó a sí mismo para cobrar dinero del seguro, Juan ejerce a la vez de agente y de paciente. Esto es lo que se conoce como una oración reflexiva. 
El hacer coincidir agente y paciente en un mismo individuo puede parecer a simple vista una cuestión simple, pero está llena de complejidades. En lenguas como el español pueden darse casos muy diversos, especialmente en cuanto al papel del supuesto agente. Conviene aclarar que en la lengua es normal distinguir entre un nivel formal, es decir, la forma, y un nivel semántico, es decir, el significado; en este tipo de construcciones, vemos a menudo lo que puede parecer una contradicción entre el nivel formal y el semántico, en casos como los siguientes:

Juan se golpeó con el mueble de la cocina.

Luis se quemó cocinando.

Estos ejemplos corresponden a lo que se conoce habitualmente como voz media. En ellos, a pesar de la apariencia reflexiva, lo normal sería entender que Luis no se quemó deliberadamente y que Juan solo sufrió el golpe, posiblemente al pasar junto al mueble, sin que él se lo "diera" a sí mismo. Es decir, estas oraciones se centran en cómo el paciente sufre los efectos de una acción, sin que haya un verdadero agente. Aunque a nivel formal parecen oraciones reflexivas, a nivel semántico no lo son. Entre los matices semánticos típicos de la voz media está el hecho de que el paciente no tenga control sobre lo sucedido; normalmente son acontecimientos que, simplemente, le sobrevienen (Kemmer, 1993; Maldonado, 2009; Langacker, 2008) ${ }^{12}$.

Obsérvese que, debido a la coincidencia formal, en español hay cierta ambigüedad en cuanto a una "lectura reflexiva" o una "lectura en voz media"13. Así, en un ejemplo como Juan se ha chocado con el coche, puede haber una interpretación reflexiva en la que Juan es agente (si él ha dirigido el coche adrede contra un muro, por ejemplo) o una interpretación en voz media, en la que Juan no ha causado la acción (si, por ejemplo, el choque ha sido debido al movimiento de otro coche).

Aunque el fenómeno es de excesiva complejidad como para abordarlo aquí de forma exhaustiva, la propuesta aquí formulada, en resumidas cuentas, es que la primera escena del sueño de la agresión se sitúa al autor a la vez en los papeles de agente (a través del YO OBSERVADOR) y de paciente (a través del YO), con lo que la escena estaría articulada siguiendo el modelo B de representación del yo. Y, de esta manera, el sueño podría estar reflejando dinámicas paralelas a las que se observan en la lengua en casos como Juan se ha chocado. Así, una posibilidad sería que la escena reprodujera una configuración similar a la de la voz media, incluyendo matices semánticos típicos de la voz media, como el hecho de que el paciente no tenga control sobre lo sucedido; y, lo que sería más llamativo, incluyendo la mencionada contradicción aparente entre el nivel formal y el nivel semántico, ya que, aunque "se ven" dos personajes, la escena gira solo en torno a uno de ellos en su calidad de paciente. De esta forma, el sueño estaría centrándose tan solo en cómo el yo recibe los efectos de la acción, sin atender a quién es el agente, como si dijera Aquella noche me di unos buenos golpes.

Y, sin descartar que pueda haber una ambigüedad similar a la que se observa en la lengua, también sería imaginable que la escena reprodujera una configuración 
más cercana a la de la reflexividad. En tal caso, quizá dentro de un planteamiento más amplio sobre la relación y sus problemas, el sueño estaría abriendo la puerta a que yo fuera el causante de sus propios males, como si el yo que sueña dijera más bien: Me he acabado propinando a mí mismo una buena paliza.

\section{Conclusiones}

Se han podido observar tres modelos que, a través de fenómenos que también quedan de manifiesto en la lengua, articulan la representación del yo en los sueños:

- Modelo A: el yo está presente únicamente a través del Yo OBSERVADOR.

- Modelo B: el yo está presente a través del yo obSERVADOR y del yo.

- Modelo C: el yo está presente a través del Yo OBSERVADOR, de un YO ${ }^{1}$ y un $\mathrm{YO}^{2}$.

Si bien dentro de la extensión de un trabajo como este tan solo es posible plantear un breve esbozo, han quedado de manifiesto algunas características claves de la representación a través de estos modelos. Una de ellas es que la selección de "actores" que representan al yo (que a veces son personajes conocidos en la realidad, y otras no) no es fruto del azar. Otra es que tampoco son casuales los atributos psíquicos y emociones que se asignan a las diferentes instancias, y también aquí se puede ver una correspondencia con la dinámica que se observa a través de la lengua. Por último, que el modo en que estas instancias interactúan entre sí pude guardar asimismo importantes paralelismos con las dinámicas que se observan en la lengua, aun las que aparentemente carecen de lógica.

En conclusión, pues, la lengua puede ser un punto de apoyo de valor inestimable a la hora de comprender la representación del yo dentro de una manifestación tan esquiva como los sueños.

\section{Notas}

1 La equiparación del sueño con la lengua, especialmente con un estadio "primitivo" o arcaico, ha sido recurrente, y va desde clásicos como Freud (1900/1991) o Fromm (1957) a autores actuales como Montangero (2013). Una de las formas más extravagante en que se ha plasmado esta intuición es, a mi entender, la obra $A$ Grammar of Dreams, de David Foulkes (1978), en el que se intenta analizar la relación entre el relato de un sueño y su contenido siguiendo los preceptos de la lingüística generativa de Chomsky.

2 Con el término inconsciente me refiero aquí al inconsciente de las ciencias cognitivas.

3 Siguiendo la convención habitual, para la notación de metáforas emplearé la fórmula [domino meta] es [dominio origen] (cf. Soriano, 2012).

4 Cf. Lakoff (1997a, 1998), de donde tomo los ejemplos citados, así como Bolognesi y Bichisecchi (2014) y Malinowski y Horton (2015); éstas ponen la metáfora en relación con los procesos de conexión propios del sueño. En los trabajos sobre sueños de terapeutas como Kramer (2017), Hartmann (2007) o Montangero (2007) también está muy presente, de un modo u otro, la identificación de metáforas Cf. asimismo Enyon (2002), que ilustra la relevancia de la metáfora en el contexto general de la terapia.

5 Cf. Lakoff (1997a, 1998), de donde tomo el ejemplo.

6 Para esta dicotomía, véase Lakoff (1997b) y Lakoff y Johnson (1999). Cf. asimismo Talmy (2015; 2000: vol.1, cap. 7), que, en el marco de su trabajo sobre dinámica de fuerzas, señala que es habitual 
que se representen las instancias de la mente como entidades en oposición (antagonist y agonist). Talmy también observa que los conceptos de superyó y ello elaborados por Freud muestran una clara analogía con estas instancias que se observan en la lengua, y propone que dichas ideas freudianas pudieron haber surgido en parte como teorización de estas nociones que forman parte de la organización lingüística. Acerca de la división del yo en construcciones reflexivas, cf. Haimann (1995).

7 En adelante se prescindirá de los términos subject y self habituales en la bibliografía, entre otras cosas por la dificultad de traducirlos de manera adecuada al castellano.

8 Acerca de los "juegos de palabras" que pueden darse en los sueños, véase Kilroe (2001).

9 Acerca de la tendencia a la hipérbole del sueño, véase Montangero (2007). Cushway y Sewell (2013) hablan de dramatización.

10 Dado que este trabajo se centra en el yo dividido, es inevitable que apenas preste atención aquí al modelo A. No obstante, esto no quiere decir que no sea frecuente; de hecho, bien podría ser el más habitual de los tres. Para otros ejemplos, me remito a Arias Oliveira (en prensa).

11 Para un análisis más completo de este sueño, teniendo el cuenta todas las partes que lo conforman, así como el contexto en sentido más amplio, me remito a Arias Oliveira (en prensa: caps. 6 y 7).

12 Para los matices semánticos de las construcciones medias, véase Kemmer (1993), Maldonado (2009) o Langacker (2008).

13 Cf. Langacker (2008).

\section{Referencias Bibliográficas}

Arias Oliveira, R. (en prensa). El sueño es lengua, la lengua es sueño. Madrid, España: Psimática.

Bolognesi, M. y Bichisecchi, R. (2014). Metaphors in Dreams: Where Cognitive Linguistics meets Psychoanalysis. Language and Psychoanalysis, 3(1), 4-22. Recuperado de: http://www.language-and-psychoanalysis.com/ article/view/1585

Cushway, D. y Sewell, R. (2013). Therapy with Dreams and Nightmares. $2^{\mathrm{a}}$ ed. Los Ángeles, LA: Sage.

Domaradzki, M. (2011). The Self in Arabic and the Relativism-Universalism Controversy. Cognitive Linguistics, $22(3), 535-567$.

Eynon, T. (2002). Cognitive linguistics. Advances in Psychiatric Treatment, 8, 399-407.

Foulkes, D. (1978). A Grammar of Dreams. Hassocks, Reino Unido: Harvester Press.

Freud, S. (1991). Die Traumdeutung. Fráncfort, Alemania: Fischer. Traducción española: La interpretación de los sueños. Traducción de L. López-Ballesteros. Madrid, España: Alianza (Trabajo original publicado en 1900).

Fromm, E. (1957). The Forgotten Language. Nueva York, NY: Grove Press.

Haiman, J. (1995). Grammatical Signs of the Divided Self. A Study of Language and Culture. En W. Abraham, T. Givón y S. Thompson (coords.), Discourse, Grammar and Typology (pp. 213-234). Amsterdam, Países Bajos: Benjamins.

Hartmann, E. (2007). The Nature and Functions of Dreaming. En D. Barrett y P. McNamara (coords.): The New Science of Dreaming, vol. 3 (pp. 171-192). Westport, NY: Praeger.

Hartmann, E. (2010). Meteorite or Gemstone? Dreaming as one end of a continuum of functioning: implications for research and for the use of dreams in therapy and self-knowledge. Dreaming, 20(3), 149-168.

Hirose, Y. (2014). The conceptual basis for reflexive constructions in Japanese. Journal of Pragmatics, 68, 99-116. Jung, C. G. (2001). “Allgemeine Gesichtspunkte zur Psychologie des Traumes”. En C. G. Jung (Ed.), Traum und Traumdeutung (pp. 89-131). Múnich, Alemania: dtv. Traducción española: "Puntos de vista generales acerca de la psicología de los sueños”. En Obra completa (vol. 8). Madrid, España: Trotta. (Trabajo original publicado en 1916)

Kemmer, S. (1993). The middle voice. Amsterdam, Países Bajos: Benjamins.

Kilroe, P. (2001). Verbal Aspects of Dreaming: A Preliminary Classification. Dreaming, 11(3), 105-113.

Kramer, M. (2017). An Approach to Understanding Dreams. Sleep Vigilance, 1, 105-113. Recuperado de: https:/ /link.springer.com/article/10.1007\%2Fs41782-017-0011-z 
Lakoff, G. (1997a). How Unconscious Metaphorical Tought Shapes Dreams. En D. Stein (coord.), Cognitive Science and the Unconscious. Washington, DC: American Psychiatric Press.

Lakoff, G. (1997b). The internal structure of the Self. En U. Neisser y D. Jopling (coords.), The Conceptual Self in Context. Culture, Experience, Self-Understanding (pp. 92-113). Cambridge, Reino Unido: Cambridge University Press.

Lakoff, G. (1998). Cómo la metáfora estructura los sueños. Revista de Psicoterapia 9(34/35), 5-30. Recuperado de: https://tienda.revistadepsicoterapia.com/como-la-metafora-estructura-los-sue-os.html

Lakoff, G. y Johnson, M. (1999). Philosophy in the Flesh. Nueva York, NY: Basic Books.

Langacker, R. (2008). Cognitive Grammar. A Basic Introduction. Oxford, Reino Unido: Oxford University Press. Lynch, D. y Frost, M. (Directores.) (1990). Twin Peaks (serie). Washington, DC: ABC.

Maldonado, R. (2009). Middle Voice as a Basic Voice System. En L. Guerrero, S. Ibáñez y V. Belloro (coords.), Studies in Role and Reference Grammar (pp. 69-111). Ciudad de México, México: Instituto de Investigaciones Filológicas, UNAM.

Malinowski, J. y Horton, C. (2015). Metaphor and hyperassociativity: the imagination mechanisms behind emotion assimilation in sleep and dreaming. Frontiers in Psychology, 6, 1132. Recuperado de: https:// www.frontiersin.org/articles/10.3389/fpsyg.2015.01132/full

Montangero, J. (2007). Comprendre ses rêves pour mieux se connaître. Paris, Francia: Odile Jacob.

Montangero, J. (2013). 40 questions et réponses sur les rêves. Paris, Francia: Odile Jacob.

Soriano, C. (2012). La metáfora conceptual. En I. Ibarretxe y J. Valenzuela (coords.), Lingüística Cognitiva (pp. 97-121). Barcelona, España: Anthropos.

Talmy, L. (2000). Toward a Cognitive Semantics. Cambridge, MA: MIT Press.

Talmy, L. (2015). Relating Language to Other Cognitive Systems: An Overview. Cognitive Linguistics, 1(1), 144. 